\title{
A INFLUÊNCIA DO FUMO NA REABILITAÇÃO COM IMPLANTES OSSEOINTEGRADOS: REVISÃO DE LITERATURA
}

\author{
Thiago Augusto Campos de MIRANDA ${ }^{1}$ \\ thiagomiranda_13@hotmail.com
}

\author{
Priscila Carneiro OLIVEIRA ${ }^{2}$ \\ carneiro_priscila@hotmail.com
}

Laís Sara EGAS 3

laissaraegas@gmail.com

Daniela PONZONI ${ }^{4}$

dponzoni@unesp.br

\author{
Roberta Catapano NAVES 5 \\ catapanonaves@hotmail.com
}

\section{RESUMO}

O fumo é um forte indicador de risco para implantes osseointegrados e os insucessos estão relacionados com as fases envolvidas durante o processo de reparo e Osseointegração, além da sobrevida dos implantes em decorrência do aumento da incidência de peri-implantite e mucosite peri-implantar, e como consequência em alguns casos a perda do implante. A nicotina representa a substância de maior expressão e toxicidade nos cigarros sobre os tecidos da cavidade oral. Assim, o presente estudo tem por objetivo revisar a literatura associando a influência e o risco do tabagismo à importância da saúde peri-implantar para previsibilidade do tratamento. Há estudos que também concluíram que o fumo aumenta o risco de peri-implantite e perda óssea peri-implantar. Assim, podemos concluir que o tabaco influencia negativamente na osseointegração e sobrevida dos implantes de titânio, devido aos efeitos citotóxicos das suas substâncias.

DESCRITORES: OSSEOINTEGRAÇÃO, TABAGISMO, PERIODONTO.

1 Estudante de Odontologia da UNIRB

2 Mestre em Periodontia, Especialista em Implantodontia, Professora da Faculdade Regional da Bahia (UNIRB)

3 Especialista em Implantodontia, Mestranda em Implantodontia da Universidade Estadual Paulista "Júlio Mesquita Filho" (UNESP)

4 Doutora em Cirurgia e Traumatologia Bucomaxilofacial, Professora Assistente da Universidade Estadual Paulista "Júlio Mesquita Filho" (UNESP)

5 Mestre em Clínica Odontológica, Especialista em Periodontia, Professora da Faculdade Regional da Bahia (UNIRB) e da Escola Bahiana de Medicina e Saúde Pública 
MIRANDA TAC, OLIVEIRA PC, EGAS LS, PONZONI D, NAVES RC. A influência do fumo na reabilitação com implantes osseointegrados: revisão de literatura. Rev. Odontol. Univ. Cid. São Paulo 2018 abr/jun 30(2) 169-76

ISSN 1983-5183

\title{
THE INFLUENCE OF SMOKING ON REHABILITATION OF OSSEOINTEGRATION IMPLANTS: A LITERATURE REVIEW
}

\begin{abstract}
Smoke is one of the main factors on the Osseointegrated implants failure. The cases of nonsuccess are related to the phases involved in the osseointegration procedure, also the survival rate of the implants diminish in occasion of increased gingivitis, peri-implantitis, consequently generating the loss of the implant. Nicotine is the most representative substance in cigarettes, thus many of the subsequent effects on the body are related to it. Along these lines the present study intends to review literature associating the influence of smoking and its effects on patients with severe generalized chronic periodontitis. The literature review showed that periodontal tissues health is very important to the osseointegration, because the cases of peri-implantitis identified in patients with previous periodontitis are common.There are also studies that concluded that smoking raises the risk of peri-implantitis and peri-implant bone loss. However, recent systematic revisions have not demonstrated significant difference in the implant failure among smokers and non-smokers. Yet, there are in literature enough scientific data that prove the negative effects of nicotine and smoking in the process of tooth repair and its many phases. Therefore, inside the limits of this study and considering the material addressed in the literature review, it is possible to conclude that tobacco has negative influence on osseointegration and the survival rate of titanium implants.
\end{abstract}

DESCRIPTORS: OSSEOINTEGRATION, TOBACCO USE DISORDER, PERIODONTIUM.

\section{INTRODUÇÃO}

A Osseointegração é definida como uma conexão estrutural e funcional direta entre osso organizado vivo e a superfície de um implante submetido à carga. Dessa forma, a criação e manutenção da osseointegração dependem da compreensão da capacidade de reparo de remodelação tecidual'.

No entanto, a evolução das pesquisas envolvendo os implantes osseointegráveis tem apresentado as influências de diferentes condições sistêmicas e locais que podem interferir durante o processo de reparo ou na sobrevida dos implantes em função². Dentre essas alterações, o fumo está diretamente associado a algumas alterações nesse processo, pois o tabaco está associado a diversos eventos nocivos à saúde oral como: aumento do risco de câncer bucal, maiores severidade e incidência de doença periodontal, menor ganho de inserção gengival após tratamento periodontal, dificuldades na reparação de enxertos ósseos, inadequada formação de coágulo sanguíneo pós-exodontias ${ }^{3}$.

Além disso, o fumo ainda diminui a vascularização local e, consequentemente, a resposta imunoinflamatória e pode, também, modificar negativamente o contato osso/implante, o preenchimento e densidade óssea devido ao seu efeito constante na proliferação dos precursores celulares e na vascularização no sítio do implante 2 . Sem deixar de citar a nicotina e seus subprodutos presentes tanto no plasma sanguíneo quanto no fluido gengival em concentrações seis vezes maiores se comparado à concentração salivar. Dessa forma, a nicotina tem sido associada a várias alterações celulares que podem contribuir para o início e posterior progressão da doença periodontal ${ }^{4}$.

Tal como ocorre nos tecidos periodontais, o tabagismo também provoca alterações nos tecidos peri-implantares: vasoconstrição, redução do fluxo sanguíneo, maior probabilidade de desenvolver periimplantite e interferência no reparo pós-operatório ${ }^{5}$. 
MIRANDA TAC, OLIVEIRA PC, EGAS LS, PONZONI D, NAVES RC. A influência do fumo na reabilitação com implantes osseointegrados: revisão de literatura. Rev. Odontol. Univ. Cid. São Paulo 2018 abr/jun 30(2) 169-76

ISSN 1983-5183

Implantes instalados em pacientes tabagistas, no decorrer dos anos, mostraram que há chance maior de insucesso na osseointegração, consequentemente levando à sua perda. Esse achado está relacionado aos efeitos nocivos que os componentes químicos do cigarro podem causar. Devido às grandes taxas de perdas de implantes em pacientes, há necessidade de haver melhor conhecimento da verdadeira influência que o hábito de fumar pode exercer na osseointegração e no tecido peri-implantar, quando comparados aos implantes colocados em pacientes não fumantes ${ }^{6}$.

Sendo assim, o presente estudo tem como objetivo revisar a literatura, associando a influência do fumo na reabilitação com implantes osseointegrados à importância da saúde peri-implantar.

\section{METODOLOGIA}

Os critérios para inclusão das citações presentes na revisão foram: artigos na íntegra, em língua inglesa e portuguesa, que abordaram o tema. Alguns dos trabalhos, Branemark et al.' (1969), Lindhe7 (1988) e dos autores Carranza e Newman ${ }^{8}$ (2006), foram usados pelo fato de serem clássicos para uma melhor compreensão e entendimento do caso, além de que são uma grande referência para os estudos atuais.

Para a construção deste trabalho foram consultados diversos artigos científicos, cuja pesquisa foi efetuada através das bases de dados: Scielo, PubMed, Bireme, Lilacs. Foram usados os seguintes descritores: osseointegração, tabagismo, periodonto, fumo. Não foi aplicado nenhum filtro de restrição de datas ou idiomas.

\section{REVISÃO DE LITERATURA}

\section{A IMPORTÂNCIA DA SAÚDE PERIODONTAL}

\section{TECIDO PERI-IMPLANTAR}

Os tecidos ao redor dos implantes são chamados de peri-implantares, são compostos de uma porção de tecido duro, osso lamelar e medular e uma porção de tecido mole, a mucosa peri-implantar. A mucosa peri-implantar saudável pode variar de aparência de acordo com as características da mucosa do rebordo onde foi instalado o implante 9 . Dessa forma, quando um implante é instalado em um rebordo que permita que mucosa queratinizada envolva o implante, a mucosa peri-implantar apresenta-se firme e com coloração rosa. O tecido peri-implantar é semelhante ao tecido periodontal, constituído de epitélio bucal queratinizado, epitélio sulcular, epitélio juncional e uma zona de tecido conjuntivo formado por fibras colágenas periimplantares ancoradas na crista óssea marginal e dispostas paralelas à superfície do implante ${ }^{10}$.

A diferença mais significante entre o tecido periodontal e a mucosa peri-implantar é a vascularização pois, no primeiro, os vasos provêm do tecido ósseo e do ligamento periodontal, enquanto no tecido periimplantar a vascularização provém diretamente do osso alveolar ${ }^{8,10}$.

O tecido peri-implantar apresenta aproximadamente $2 \mathrm{~mm}$ de epitélio juncional e de 1 a $1,5 \mathrm{~mm}$ de tecido conjuntivo, devido à ausência de cemento na estrutura, as fibras colágenas posicionam-se paralelas ao implante ${ }^{11}$. A composição do tecido conjuntivo ao redor do implante difere da composição do tecido conjuntivo ao redor do dente pelo seu maior conteúdo de fibras colágenas e menor conteúdo de células e vasos sanguíneos ${ }^{9}$.

PERI-IMPLANTITE

A peri-implantite é definida como um processo inflamatório que afeta os tecidos ao redor de 
MIRANDA TAC, OLIVEIRA PC, EGAS LS, PONZONI D, NAVES RC. A influência do fumo na reabilitação com implantes osseointegrados: revisão de literatura. Rev. Odontol. Univ. Cid. São Paulo 2018 abr/jun 30(2) 169-76

ISSN 1983-5183

um implante osseointegrado, resultando em perda do osso de suporte. Representa uma infecção sítioespecífica, com diversos padrões em comum com a periodontite crônica. A peri-implantite é diagnosticada quando ocorre perda progressiva de osso peri-implantar, excedendo os limites de tolerância de reabsorção de osso, após sucesso da osseointegração do implante, ou seja, a média de perda óssea vertical ao redor do implante não deve ser maior que $0,2 \mathrm{~mm}$ anualmente ${ }^{12}$. A literatura aponta que os principais sinais da periimplantite são: inflamação da mucosa peri-implantar, hiperemia, sangramento à sondagem, profundidade de sondagem $>4 \mathrm{~mm}$, presença ou não de supuração e coleção purulenta além da perda de inserção clínica e perda óssea peri-implantar progressiva observada radiograficamente, podendo levar até à perda total de osteointegração ${ }^{13}$. A peri-implantite pode ser reversível em alguns casos se houver intervenção cirúrgica ${ }^{14}$.

\section{FUMO E OSSEOINTEGRAÇÃO}

O fumo constitui um indicador de risco associado à peri-implantite; o hábito de fumar provoca alterações nos tecidos peri-implantares como: vasoconstrição, redução do fluxo sanguíneo e da neoangiogênese e consequentemente interferindo no reparo pós-cirúrgico. A fumaça do cigarro apresenta mais de quatro mil substâncias tóxicas conhecidas, incluindo monóxido de carbono, cianeto de hidrogênio, radicais oxidantes reativos, um grande número de carcinógenos e a principal molécula psicoativa e que leva ao vício - a nicotina ${ }^{10,15}$.

A nicotina tem efeito na síntese das proteínas celulares e prejudica a habilidade dos fibroblastos gengivais na adesão e proliferação. Além disso, aumenta a adesividade plaquetária, produz vasoconstricção cutânea e interfere na morfologia da microcirculação, gerando isquemia dos tecidos, a qual resulta muitas vezes em necrose $\mathrm{e}^{10,16}$.

A inalação da fumaça de cigarro influencia negativamente a densidade do osso preexistente e a qualidade do osso neoformado ao redor de implantes de titânio, menor contato osso-implante e preenchimento das roscas, afetando tanto osso cortical quanto esponjoso e a absorção de nicotina afeta em especial o osso esponjoso. Tanto a interrupção temporária quanto a definitiva promovem um efeito positivo no osso ao redor dos implantes de titânio ${ }^{16,17}$.

Em estudo realizado com controle radiográfico, os pacientes foram divididos em 3 grupos: não fumantes, fumantes e fumantes pesados (considerado fumante pesado o indivíduo que fuma 20 ou mais cigarros). 0 acompanhamento radiográfico foi realizado por um período de 1 a 7 anos após a colocação dos implantes. Foram analisados 646 implantes instalados em 161 pacientes. Os resultados revelaram uma maior perda óssea marginal nos fumantes com maior incidência em nível do maxilar superior ${ }^{17}$. Foram observadas diferenças entre fumantes moderados e pesados, sendo estes últimos mais frequentemente associados ao insucesso dos implantes. A única exceção desse achado foi a inexistência de uma diferença estatisticamente significativa na taxa de sucesso dos implantes colocados na mandíbula, quando se comparavam os fumantes com os não fumantes. Os autores observaram, ainda, que a prevalência de osso tipo IV é duas vezes maior nos fumantes pesados do que nos não fumantes ou até nos fumantes leves ${ }^{18}$.

Existe associação do tabaco com perda óssea alveolar e periodontite, confirmando o efeito prejudicial do fumo no controle da saúde periodontal. Muitos procedimentos cirúrgicos como a regeneração tecidual guiada e a colocação de implantes estariam, dessa forma, contraindicados ${ }^{10}$.

Deluca et al. ${ }^{19}$ (2006) avaliaram a taxa de sobrevida de implantes dentais em 464 pacientes ao longo de 10 anos. A Taxa global de falha dos implantes foi de 7,72\%. Os pacientes fumantes e que receberam 
MIRANDA TAC, OLIVEIRA PC, EGAS LS, PONZONI D, NAVES RC. A influência do fumo na reabilitação com implantes osseointegrados: revisão de literatura. Rev. Odontol. Univ. Cid. São Paulo 2018 abr/jun 30(2) 169-76

ISSN 1983-5183

implantes tiveram alta taxa de falha de osseointegração $(23,08 \%$,$) quando comparados aos pacientes não$ fumantes (13.33\%), relatando que o cigarro é um fator de risco no aumento de falhas nos implantes ${ }^{19}$.

Jones e Triplett $^{20}$ (1992) compararam 15 pacientes (10 não fumantes e 5 fumantes) submetidos a cirurgias para enxerto autógeno com colocação simultânea de implantes para investigar os efeitos negativos do cigarro sobre a cicatrização. Os cinco fumantes tiveram a cicatrização prejudicada, com perda do implante e/ou de osso; quatro deles (80\%) admitiram ter fumado no período pré-operatório, sendo que em apenas um (10\%) dos 10 não fumantes a cicatrização foi problemática. 0 papel de outros potenciais fatores como a idade, o sexo e nutrição não pode ser avaliado devido ao número limitado de casos, mas os autores concluíram que há uma forte evidência clínica que implica o hábito de fumar cigarros como grande risco aos pacientes submetidos a esses procedimentos ${ }^{20}$.

Acredita-se, também, que cessar o fumo uma semana antes do procedimento cirúrgico e continuar sem fumar por mais 8 semanas após a instalação dos implantes diminuem os índices de falhas de implantes nos pacientes fumantes, tornando-os similares aos dos pacientes não fumantes ${ }^{20}$.

Entretanto, o tabaco não pode ser considerado contraindicação absoluta para a reabilitação com implantes dentais, ainda que haja maior tendência ao risco de perda precoce de implantes dentais, o que deve ser informado e bem esclarecido ao paciente tabagista, principalmente sobre uso do cigarro no período inicial do reparo tecidual. Além disso, independente da cessão do ato de fumar, imediatamente, antes da cirurgia de instalação dos implantes, aqueles que fazem uso do cigarro por longos períodos apresentam maior índice de perda óssea marginal tardia ${ }^{19}$.

\section{DISCUSSÃO}

Os fatores envolvidos com a obtenção de sucesso na osseointegração são diversos e incluem biocompatibilidade do material, macro e microestrutura dos implantes de alto desempenho, técnica cirúrgica apropriada, boa qualidade e quantidade óssea e condições favoráveis de carga. Da mesma maneira numerosos fatores associados a falhas dos implantes dentais têm sido descritos: osso pobre, técnica cirúrgica traumática, sobrecarga oclusal, estrutura do implante desfavorável, condições sistêmicas, tabaco e uso de outras drogas $^{15,16,21}$.

Pacientes fumantes costumam ter uma pior higienização bucal, maior consumo de álcool e, portanto, efeitos adversos diferentes sobre o aspecto odontológico. O tabaco e, mais especificamente, a nicotina, droga mais nociva à saúde e universalmente consumida ${ }^{22}$, têm sido estudados como grandes intervenientes no fracasso da osteointegração dos implantes dentários, devido aos seus efeitos na cavidade oral ${ }^{23}$. Estes podem ser expressos tanto sistêmicos quanto localmente. A nicotina causa, entre outros efeitos, vasoconstrição periférica, isquemia tecidual e diminui a tensão de oxigênio ${ }^{21}$. Para amenizar as complicações em pacientes fumantes devemos lançar mão de tecnologias (implantes de superfície tratada) e técnicas (protocolo de

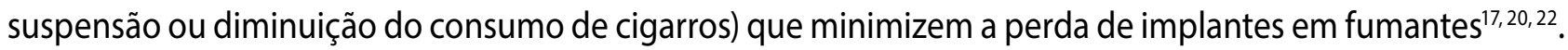

Há evidências clínicas crescentes de que o fumo tem um efeito nocivo na progressão da doença periodontal e na cicatrização após a terapia periodontal. As seguintes alterações em fumantes podem ser encontradas: mudanças na coloração da estrutura dentária e gengival; leucoplasia gengival; estomatite nicotínica; retardo da cicatrização; aumento transitório no fluxo de líquido gengival; gengivite e periodontite mais graves ${ }^{8}$.

Além disso, é necessária a preservação da maior quantidade de osso alveolar para instalação de 
MIRANDA TAC, OLIVEIRA PC, EGAS LS, PONZONI D, NAVES RC. A influência do fumo na reabilitação com implantes osseointegrados: revisão de literatura. Rev. Odontol. Univ. Cid. São Paulo 2018 abr/jun 30(2) 169-76

ISSN 1983-5183

implantes dentários. Com isso, a colocação de implantes logo após a exodontia tem sido muito discutida nos últimos anos, devido às falhas clínicas persistentes e também à perda óssea vestibular causada pela própria exodontia. Sendo assim, para superar esses desafios são preconizadas cirurgias minimamente invasivas, como auxílio do periótomo ${ }^{24}$. Fato ratificado pelos estudos que afirmam que o periótomo, na maioria das vezes, alcança as fibras do terço cervical e médio, separando o ligamento periodontal do dente, de forma que a cirurgia se torne menos traumática ${ }^{25}$. Ainda, o tabagismo associado ao controle de biofilme dental insuficiente e o posicionamento inadequado dos implantes são os fatores principais para a perda óssea ${ }^{26}$.

Em relação ao consumo de cigarros, ainda são comuns, entre os pacientes fumantes, maior possibilidade de acúmulo de biofilme, incidência de gengivite e periodontite, maior taxa de perda dentária e reabsorção do rebordo. $O$ consumo de cigarros afeta adversamente a cicatrização e pode colocar em risco o sucesso de enxertos e de implantes. Afirmaram alguns estudos que a taxa de perda óssea marginal ao redor dos implantes é cerca de três vezes mais alta em indivíduos fumantes e, ainda, que a incidência de complicações pós-cirúrgicas é maior entre os fumantes ${ }^{27}$. Além disso, o efeito prejudicial do tabaco no processo de reparo dos tecidos devido ao envolvimento com a revascularização pode comprometer a cicatrização óssea após a colocação de um implante, podendo inclusive diminuir a taxa de sobrevivência dos tecidos ${ }^{21,23}$. Notou-se, também, uma percentagem de falhas de $11,28 \%$ em fumantes para $4,76 \%$ em não fumantes, exceto para a área posterior da mandíbula. Na maxila de fumantes encontrou-se um índice extremamente alto de falhas para implantes menores, excedendo a percentagem de $25 \%$ proposta como o máximo aceitável. Observou-se a relação da qualidade óssea e fumo na incidência de falhas de implantes. Foi encontrada uma incidência significativamente maior de osso tipo 4 em fumantes moderados e pesados (37,9\%) em relação a não-fumantes e fumantes leves $(17,6 \%)$ e os autores concluíram que fumantes pesados e moderados têm uma influência negativa na qualidade óssea, devendo ser avisados quanto ao prognóstico comprometido em relação aos implantes dentais ${ }^{18}$.

Em estudo com seio maxilar foi realizada abertura pela técnica Cadwell-Luc, que é uma técnica cirúrgica indicada quando a altura óssea residual não permite a instalação dos implantes de comprimento standard ou o uso de técnicas de elevações menores como a técnica de Summers com o uso dos osteótomos próprios. A instalação dos implantes poderá ser feita numa fase cirúrgica seguida da elevação do seio maxilar, obrigando a existência de uma altura mínima óssea de $5 \mathrm{~mm}$, ou em 2 fases: elevação do seio maxilar e posteriormente instalação dos implantes, geralmente utilizada em alturas ósseas compreendidas entre $1 \mathrm{~mm}$ e $4 \mathrm{~mm}$; quando não atingimos a estabilidade primária do implante ${ }^{28}$.

Em relação à suspensão do fumo pré e pós-operatório, observou-se uma vez que a abolição do fumo 1 semana antes e 8 semanas após a cirurgia influenciou positivamente na diminuição da taxa de insucesso dos implantes nos fumantes. Além disso, os fumantes que aceitaram a prescrição do regime de abstenção de uma semana antes e oito semanas depois tiveram índices de falhas similares aos dos não fumantes, indicando que a cessação do hábito, mesmo que por curto tempo, resultou em significativa melhoria no índice de sucesso dos implantes ${ }^{29}$.

\section{CONCLUSÃO}

Pode-se observar que não há contraindicação absoluta para a instalação de implantes osteointegrados em pacientes tabagistas, porém, tal hábito é nocivo e deve ser levado em consideração durante o planejamento reabilitador. Para o sucesso do tratamento em pacientes com hábitos tabagistas, é fundamental o esclarecimento, antes do procedimento, sobre as possíveis falhas na osseointegração. 
MIRANDA TAC, OLIVEIRA PC, EGAS LS, PONZONI D, NAVES RC. A influência do fumo na reabilitação com implantes osseointegrados: revisão de literatura. Rev. Odontol. Univ. Cid. São Paulo 2018 abr/jun 30(2) 169-76

ISSN 1983-5183

\section{REFERÊNCIAS}

1. BRÄNEMARK PI, Adell R, Breine U, Hansson BO, Lindstrom J, Ohlsson A. Intra-osseous anchorage of dental prostheses. I. Experimental studies. Scandinavian journal of plastic and reconstructive surgery 1969 3(2):81-100.

2. ALMEIDA JM, Altomani AC, Matheus HR, Novaes VCN, Faleiros PL, Braite MA. Influêcia do fumo na osseointegração dos implantes de titânio. Braz J Periodontol 2015 Set.;25(3):3540.

3. GOURGIOTIS S, Aloizos S, Aravosita P, Mystakelli C, Isaia EC, Gakis C, et al. The effects of tobacco smoking on the incidence and risk of intraoperative and postoperative complications in adults. The surgeon : journal of the Royal Colleges of Surgeons of Edinburgh and Ireland 2011 Aug;9(4):225-32.

4. CARVALHO PSP, Rosa AL, Bassi APF, Pereira LAVD. Biomateriais aplicados a implantodontia. ImplantNews 2010 mai./jun. ;7(3a-PBA):56-65.

5. DAUD SLM. A influência do tabagismo no insucesso dos tratamentos odontológicos [Dissertação]. São Paulo: Universidade de São Paulo, Faculdade de Odontologia; 2003.

6. PETKOV A. Osteointegração em pacientes tabagistas: revisão de literatura [Monografia de Graduação]. Florianópolis, SC: Universidade Federal de Santa Catarina, Centro de Ciências da Saúde, Odontologia; 2013.

7. LINDHE J. Tratado de periodontologia clínica. Rio de Janeiro: Guanabara Koogan; 1988.

8. CARRANZA Jr. FA, Newman MG. Periodontia clínica. 12. ed. Rio de Janeiro: Guanabara Koogan; 2016.

9. ARAÚJO M, Lubiana NF. Caracterísiticas dos tecidos peri-implantares. Periodontia 2008 18(4):8-13.

10. PREBER H, Bergstrom J. Effect of cigarette smoking on periodontal healing following surgical therapy. Journal of clinical periodontology 1990 May;17(5):324-8.

11. FARIAS LSF, Oliveira RA, Gomes TN. Prevalência de peri-implantite: revisão de literatura. Rev Interfaces 2016 abr.;3(9):65-73.

12. ROMEIRO RL, Rocha RF, Jorge AOC. Etiologia e tratamento das doenças peri-implantares. Odonto 2010 jul./dez. ;18(36):59-66.

13. SANTIAGO Junior JF, Lemos CAA, Batista VES, Mello CC, Almeida DAF, Verri FR, et al. Manutenção em próteses implantossuportadas: peri-implantite. Rev Odontol Araçatuba 2013 34(2):14-22.

14. FAVERANI LP, Ferreira GR, Gaetti-Jardim EC, Okamoto R, Shinohara EH, Assunção WG, et al. Implantes osseointegrados: evolução e sucesso. Rev Salusvita 2011 30(1):47-58.

15. ESPOSITO M, Hirsch JM, Lekholm U, Thomsen P. Biological factors contributing to failures of osseointegrated oral implants. (I). Success criteria and epidemiology. European journal of oral sciences 1998 Feb;106(1):527-51. 
MIRANDA TAC, OLIVEIRA PC, EGAS LS, PONZONI D, NAVES RC. A influência do fumo na reabilitação com implantes osseointegrados: revisão de literatura. Rev. Odontol. Univ. Cid. São Paulo 2018 abr/jun 30(2) 169-76

ISSN 1983-5183

16. ZAVANELLI RA, Guilherme AS, Castro AT, Fernandes JMA, Pereira RE, Garcia RR. Fatores locais e sistêmicos relacionados aos pacientes que podem afetar a osseointegração. RGO 2011 Abr.-Jun. ;59((supl.1)):133-46.

17. NITZAN D, Mamlider A, Levin L, Schwartz-Arad D. Impact of smoking on marginal bone loss. The International journal of oral \& maxillofacial implants 2005 Jul-Aug;20(4):605-9.

18. BAIN CA, Moy PK. The association between the failure of dental implants and cigarette smoking. The International journal of oral \& maxillofacial implants 1993 8(6):609-15.

19. DELUCA S, Habsha E, Zarb GA. The effect of smoking on osseointegrated dental implants. Part I: implant survival. The International journal of prosthodontics 2006 Sep-Oct;19(5):491-8.

20. JONES JK, Triplett RG. The relationship of cigarette smoking to impaired intraoral wound healing: a review of evidence and implications for patient care. Journal of oral and maxillofacial surgery : official journal of the American Association of Oral and Maxillofacial Surgeons 1992 Mar;50(3):237-9; discussion 9-40.

21. HADI SA, Ashfaq N, Bey A, Khan S. Biological factors responsible for failure of osseointegration in oral implants. Biol Medicine 2011 3(2):164-70.

22. FEITELSON JB, Rowell PP, Roberts CS, Fleming JT. Two week nicotine treatment selectively increases bone vascular constriction in response to norepinephrine. Journal of orthopaedic research : official publication of the Orthopaedic Research Society 2003 May;21(3):497-502.

23. POCZTARUK RL, Zaparoli ACR, Ballardin L, Rivaldo EG, Frasca LCF, Fernandes EL. Osseointegração peri-implantar em pacientes fumantes. Stomatos 2006 jul.-dez.;12(23):

24. SAUND D, Dietrich T. Minimally-invasive tooth extraction: doorknobs and strings revisited! Dental update 2013 May;40(4):325-6, 8-30.

25. DYM H, Weiss A. Exodontia: tips and techniques for better outcomes. Dental clinics of North America 2012 Jan;56(1):245-66, x.

26. PAIVA J, Almeida R. Atuação clínica baseada em evidências científicas. São Paulo: Artes Médicas; 2005.

27. LEVIN L, Schwartz-Arad D. The effect of cigarette smoking on dental implants and related surgery. Implant dentistry 2005 Dec;14(4):357-61.

28. CORREIA F, Faria Almeida R, Lemos Costa A, Carvalho J, Felino A. Levantamento do seio maxilar pela técnica da janela lateral: tipos enxertos. Rev Portuguesa Estomatologia, Medicina Dentária e Cirurgia Maxilofacial 2012 2012/07/01/;53(3):190-6.

29. BAIN CA. Smoking and implant failure--benefits of a smoking cessation protocol. The International journal of oral \& maxillofacial implants 1996 Nov-Dec;11(6):756-9.

RECEBIDO EM 01/12/2017

ACEITO EM 04/04/2018 